\title{
Focal Post Traumatic Rhabdomyolysis of Thigh Muscle - USG and CT Imaging
}

\author{
Prerna Anup Patwa ${ }^{1}$, Suresh Vasant Phatak ${ }^{2}$, Monika Pote ${ }^{3}$, Rajabala Pradeep Dhande ${ }^{4}$
}

${ }^{1}$ Department of Radiodiagnosis, DMIMS, Sawangi (Meghe), Wardha, Maharashtra, India. ${ }^{2}$ Department of Radiodiagnosis, DMIMS, Sawangi (Meghe), Wardha, Maharashtra, India. ${ }^{3}$ Department of Radiodiagnosis, DMIMS, Sawangi (Meghe),

Wardha, Maharashtra, India. ${ }^{4}$ Department of Radiodiagnosis, DMIMS, Sawangi (Meghe), Wardha, Maharashtra, India.

\section{INTRODUCTION}

Rhabdomyolysis as a term, literally means striated muscle breakdown, where these lysed cells of the muscles produce substances which when released causes severe damage to the body. And to diagnose this medical condition is where the art of imaging comes into picture. And hence, we are presenting a case report on rhabdomyolysis showing distinct ultrasound and CT features.

Rhabdomyolysis is the skeletal muscle necrosis with liberation of intracellular substances of the lysed cell into the extracellular fluid and circulation. These substances are electrolytes, purines, enzymes (such as creatine kinase) and myoglobin, which may cause further life-threatening complications.1,2 Diffuse rhabdomyolysis caused due to over-exertion is found in athletes, while focal rhabdomyolysis is rare. Early diagnosis along with its swift management is thus crucial to prevent its complications and Imaging can be a complementary part of the detection and assessment of the extent of rhabdomyolysis. Despite the fact that, the definite diagnosis depends on laboratory tests, imaging studies are usually one of the first modalities used to examine patients. Drugs and other toxic agents, infections, physical exertion, crush injury, and muscle disease are some of the common causes of rhabdomyolysis. And it typically present as muscle fatigue, pain, weakness, and tea-coloured urine. Although in the initial stage of the disease, these clinical symptoms may be subtle and so it is easily missed ${ }^{2}$. The causes for rhabdomyolysis maybe many, but they often lead to a final common feature, which is muscle tissue breakdown causing myocyte destruction and release of its components into the circulatory system. All these finally lead to muscle ischemia and cell breakdown. Rhabdomyolysis may occur due to traumatic events and the pathophysiology actually takes place once pressure is relieved from the damaged tissue, and the necrotic muscles release their components into circulation. ${ }^{1}$

Muscle ischemia caused by traumatic events interferes with oxygen delivery to the cells, limiting the production of ATP. If oxygen deprivation is maintained for prolonged periods this may result in muscle cell necrosis. Skeletal muscle ischemia may result from either localized or generalized conditions. Localized causes include compression of blood vessels. ${ }^{3}$
Corresponding Author: Dr. Suresh V Phatak,

Acharya Vinoba Bhave Rural Hospital, DMIMS, Sawangi (Meghe), Wardha, Maharashtra, India.

E-mail: suresh_phatak@yahoo.com

DOI: $10.14260 /$ jemds/2020/558

How to Cite This Article:

Patwa PA, Phatak SV, Pote M, et al. Focal post traumatic rhabdomyolysis of thigh muscle USG and CT imaging. J Evolution Med Dent Sci 2020;9(35):2571-2573, DOI: $10.14260 / \mathrm{jemds} / 2020 / 558$

Submission 04-05-2020,

Peer Review 14-07-2020,

Acceptance 22-07-2020,

Published 31-08-2020.

Copyright (C) 2020 JEMDS. This is an open access article distributed under Creative Commons Attribution License [Attribution 4.0 International (CC BY 4.0)]

\section{PRESENTATION OF CASE}


A 22-year-old young male was brought to the emergency department with history of trauma over both lower limbs particularly thigh. There was a history of heavy object falling over the legs. He was managed conservatively and consequently discharged after 4 days. After a week he visited the hospital again and now he presented with loss of limb movements since a day, complains of chest pain and breathlessness. He was in distress and confused. On physical examination, vital signs were within normal limits but there was induration [Figure 1] and loss of sensitivity in the right thigh region with no sensory deficits. The skin over that region was warm and dry and he had pigmented urine. His laboratory findings were remarkable, as follows: creatine kinase (CK) $1600 \mathrm{U} / \mathrm{L}$, creatinine (Cr) $16.2 \mathrm{mg} / \mathrm{dL}$, and urea $257 \mathrm{mg} / \mathrm{dL}$, ALT 717 U / L, AST 236 U / L, ALP 220 U / L. Raised total bilirubin of $11.2 \mathrm{mg} / \mathrm{dL}$ and slightly decreased serum calcium $6.2 \mathrm{mg} / \mathrm{dL}$. Ultrasonography of the local site was performed which showed the following findings: ground glass-like or cloudy image of reduced echogenicity [Figure 2], hyperechoic intramuscular areas in the muscles, irregular anechoic areas in the muscle with irregular and heterogeneous muscle fibres (muscular disorganisation) [Figure 3] and no blood flow signals compatible with fluid [Figure 4]. The patient underwent CT scan where the muscle showed multiple intramuscular heterogenous subtle hyper densities in the right thigh muscle suggestive of soft calcification secondary to rhabdomyolysis [Figure 5]. A presumptive diagnosis was made of rhabdomyolysis of the right thigh, due to the presence of indurations of the skin with loss of sensitivity, levels up to 5 times greater than reference CPK level with pigmented urine and acute renal function disturbance and peculiar ultrasonography finding.
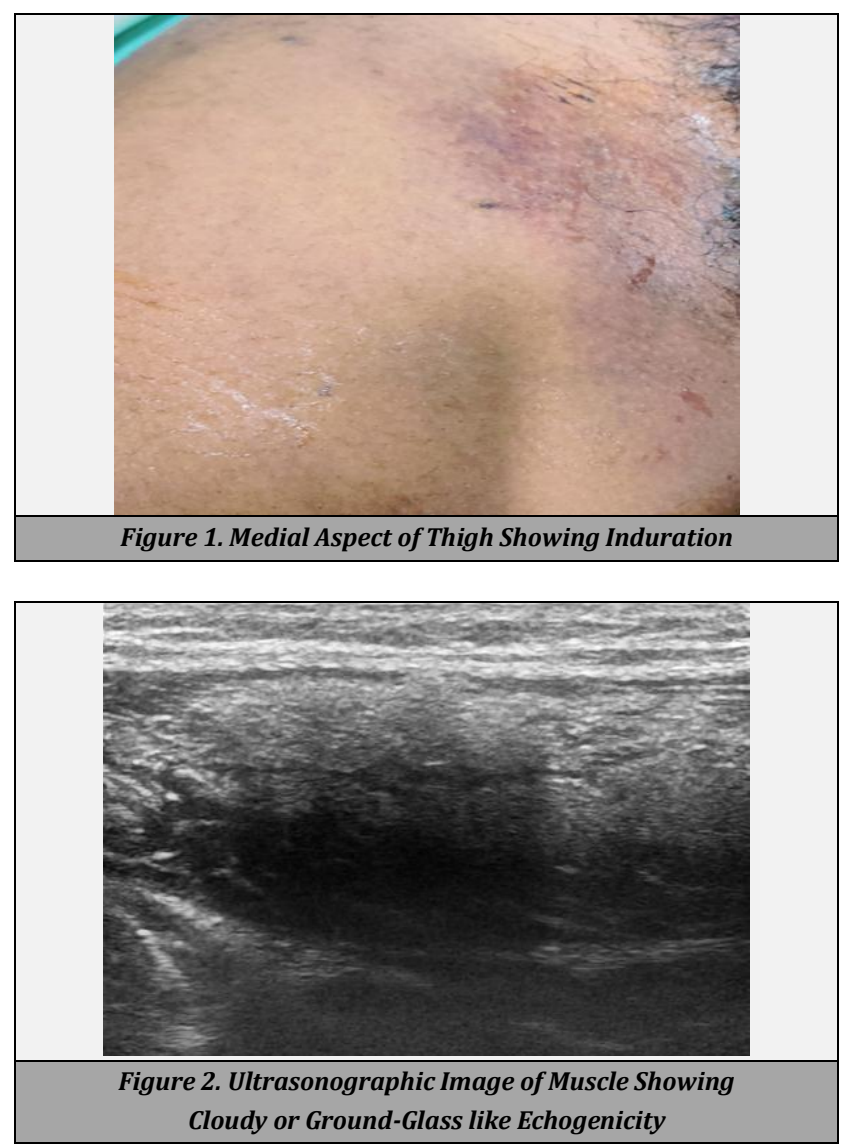
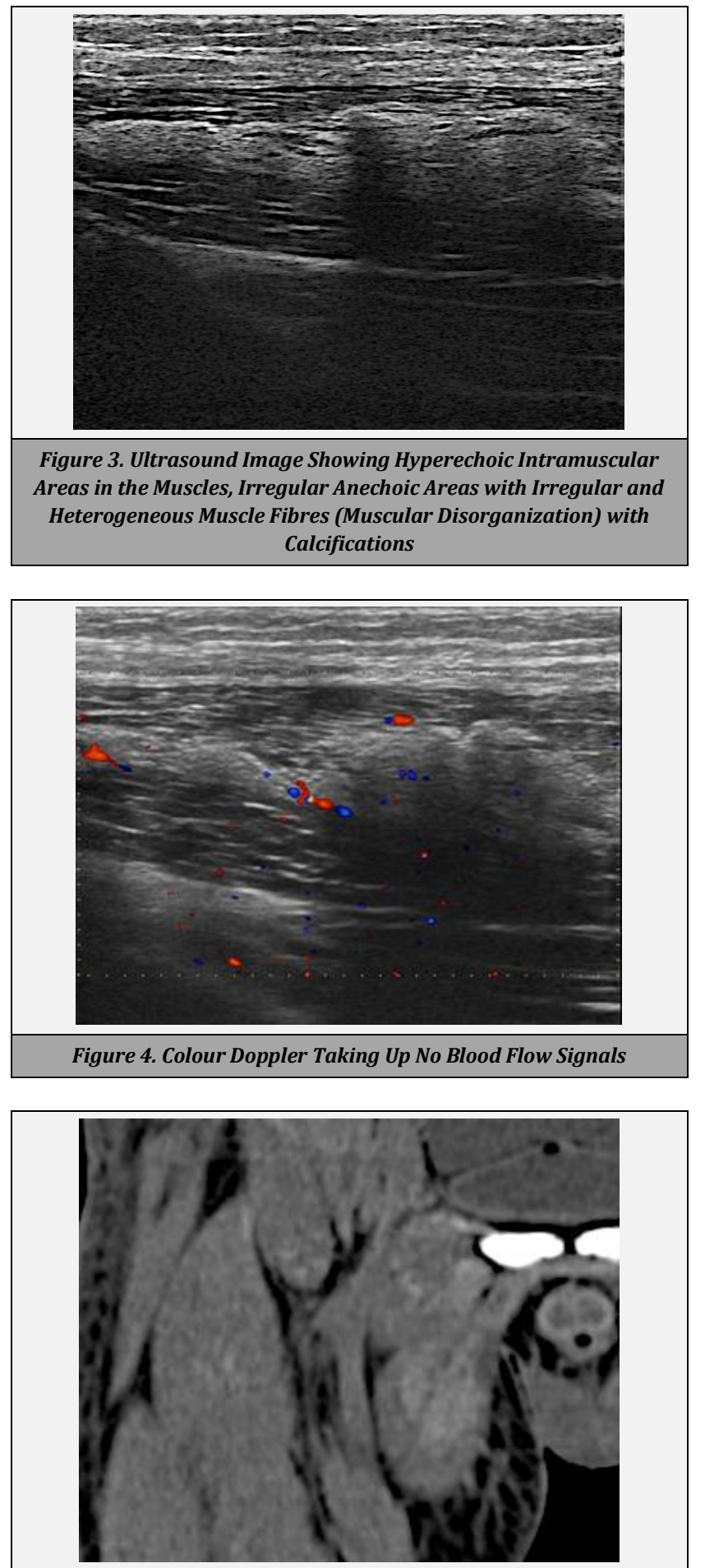

Figure 5. CT Scan Image Showing Multiple Intra-Muscular Heterogenous Subtle Hyperdensity in the Right Thigh Muscle

\section{DISCUSSION}

Rhabdomyolysis has characteristic clinical, laboratory and radiologic features, but does require a high index of suspicion 1 . And hence, early diagnosis is often difficult owing to the subtle clinical presentation. Signs on ultrasound, which are seen are reduction in echogenicity (ground glass-like or cloudy image), muscular disorganisation; increased diameter of the muscle fascia, intramuscular hyperechoic areas, uneven anechoic areas in the muscular and intramuscular periphery, with no 
signals of blood flow compatible with oedema, and normal vascularisation with preservation of waves and flow velocities. ${ }^{4}$ It is noted that mechanical compression of the muscle is etiologically important in patients with rhabdomyolysis and there are a variety of causes acting synergistically which include massive deposition of calcium in injured muscles, hyper-phosphataemia, and decreased production of renal 1,25-hydroxycholecalciferol. Now, this forms the basis of the CT findings in rhabdomyolysis which includes skeletal muscle hyperdensity corresponding to calcification. It was also noted that this finding was correlated with hypocalcaemia and renal insufficiency. The cause of the calcification is although still unknown but it is probably because phosphate separates from damaged muscle fiber and unites with serum calcium which results in a deposition. ${ }^{5}$ As compared with other imaging modalities, MRI has been shown to be the best for imaging rhabdomyolysis. Some features that are seen on MRI are increased signal intensity in the affected muscles on $\mathrm{T} 2$-weighted images. The high signal intensity that is noted on T2-weighted images could reflect transiently increased interstitial water content due to necrotic, oedematous or infarcted muscles in rhabdomyolysis. Some small low signal foci on T2-weighted images are also described in affected muscles and could be due to deposition of hemosiderin after haemorrhage. The extent of the lesion can easily be determined on an MRI scan; however, MRI is expensive, time-consuming and not easily available and hence, ultrasound is a feasible inexpensive imaging modality ${ }^{6}$. One of the other investigations that can be performed is muscle biopsy and it would reveal calcifications in the muscle fibers with the injured muscle fibers showing necrotic, oedematous, and atrophic with fatty tissue infiltration. ${ }^{5}$

\section{CONCLUSIONS}

So, rhabdomyolysis should be diagnosed at an early stage not only to prevent it's deleterious effects on various organ systems, but also to prevent complications such as, hypovolaemia, compartment syndrome, arrhythmias, disseminated intravascular coagulation, hepatic dysfunction and acute renal failure. Ultrasound in rhabdomyolysis provides necessary insights for the diagnosis, treatment and follow-up of patients with this disorder. It is an easy-to-use technological tool that can be used at the patient's bedside. CT scan is an inconstant but assertive imaging modality for diagnosis of rhabdomyolysis.

Financial or Other Competing Interests: None.

\section{REFERENCES}

[1] Keltz E, Khan FY, Mann G. Rhabdomyolysis. The role of diagnostic and prognostic factors. Muscles Ligaments Tendons J 2014;3(4):303-12.

[2] Chiu YN, Wang TG, Hsu CY, et al. Sonographic diagnosis of rhabdomyolysis. J Med Ultrasound 2008;16(2):158-62.

[3] Khan FY. Rhabdomyolysis: a review of the literature. Neth J Med 2009;67(9):272-83.

[4] Carrillo-Esper R, Galván-Talamantes Y, Meza-Ayala CM, et al. Ultrasound findings in rhabdomyolysis. Cir 2016;84(6):518-22.

[5] Nakanishi K, Shimamoto S, Kishi M, et al. CT, MR imaging and muscle biopsy in severe crush injury. Acta Radiol 1997;38(5):903-6.

[6] Lamminen AE, Hekali PE, Tiula E, et al. Acute rhabdomyolysis: evaluation with magnetic resonance imaging compared with computed tomography and ultrasonography. Br J Radiol 1989;62(736):326-30. 